\title{
Invited Discussion on: "Breast Reconstruction with SIEA Flaps: An Alternative in Selected Cases"
}

\author{
Dana Mihaela Jianu ${ }^{1}$
}

Received: 14 January 2020/ Accepted: 17 January 2020/Published online: 29 January 2020

(C) Springer Science+Business Media, LLC, part of Springer Nature and International Society of Aesthetic Plastic Surgery 2020

Level of Evidence $V$ This journal requires that authors assign a level of evidence to each article. For a full description of these Evidence-Based Medicine ratings, please refer to the Table of Contents or the online Instructions to Authors www.springer.com/00266.

The paper on breast reconstruction with SIEA flaps is well written, includes a comprehensive video, and is well conceived. The authors bring their expertise and experience of trained surgeons to the one already existing.

However, I suggest some corrections for better organization and structure so that the scientific work will be clearer, easy to comprehend and attractive:

1. A brief anatomical reminder would be welcomed.

2. In "Methods", separate the preoperative preparation and planning and postoperative care and monitoring (what equipment is used) from the surgical technique.

The surgical technique: (a) has to include for rapid visualization a scheme of the intraoperative algorithm of the authors regarding the strategy to choose the best pedicle/operation function of findings and (b) to mention the de-epithelization step in the timeline of the operation.

3. The selection of patients-as part of the title and a pillar of the work-is insufficiently exposed and structured.

4. Discussion-advantages, criteria of including and excluding SIEA flap in breast reconstruction.

Dana Mihaela Jianu

djianu02@gmail.com

1 ProEstetica Medical Center, Tudor Stefan Street, nr 38-40, Sector 1, 011658 Bucharest, Romania

\section{Compliance with ethical standards}

Conflict of interest The author declares that she has no conflict of interest.

Statement of Human and Animal Rights or Ethical Approval This article does not contain any studies with human participants or animals performed by the author.

Informed Consent For this type of study informed consent is not required.

Publisher's Note Springer Nature remains neutral with regard to jurisdictional claims in published maps and institutional affiliations. 